\title{
Efeito de um programa escolar de estimulação motora sobre desempenho da função executiva e atenção em crianças
}

\author{
Effect of school-based motor stimulation intervention on executive \\ function and attention performance in children
}

\author{
C.M. Cardeal, L.A. Pereira, P.F. Silva, N.M. França
}

ARTIGO ORIGINAL | ORIGINAL ARTICLE

\begin{abstract}
A influência da atividade física escolar no desenvolvimento da função executiva infantil ainda não é consenso entre os estudos já realizados, portanto esse estudo tem como objetivo verificar o efeito da estimulação motora, nas respostas da função cognitiva de crianças na faixa etária de 6 a 10 anos, de escolas públicas do Distrito Federal, Brasil. Foram formados 2 grupos controle $(n=40)$ e experimental $(n=40)$, avaliados antes e depois da intervenção, as variáveis analisadas: motricidade, função executiva, tempo de reação e atenção seletiva. A intervenção ocorreu durante 7 meses com aulas de educação física escolar. Os resultados demonstraram que ocorreu uma diferença significativa entre os grupos nos testes de função executiva $\left[\mathrm{F}_{(1,118)}=13.768 ; p=.001\right]$, tempo de reação $\left[\mathrm{F}_{(1,118)}=18.352 ; p=.001\right]$ e atenção seletiva $\left[\mathrm{F}_{(1,64)}=14.531 ; p=.001\right]$. Desse modo, foi observado que, o grupo que sofreu intervenção melhorou não somente o aspecto motor, mas também melhorou de forma significativa o desempenho das funções cognitivas testadas.

Palavras-chave: função executiva, desenvolvimento infantil, escolares, educação física
\end{abstract}

ABSTRACT

The influence of physical activity in children's development of executive function is not yet consensus among previous studies. So this study aims to evaluate the effects of school based motor stimulation in cognitive function responses in children aged 6 to 10 years of public schools at Brasília-Federal District, Brazil. It was formed two groups control $(n=40)$ and experimental $(n=40)$ who were studied before and after the intervention. The variable analyzed was: motor skills, executive function, reaction time and selective attention. The intervention occurred during 7 months, two times week, in a 50 minutes physical education classes. The results showed that there was a significant difference between groups in tests of executive function $\left[\mathrm{F}_{(1,118)}=13.768, p=.001\right]$, reaction time $\left[\mathrm{F}_{(1,118)}=18.352, p=.001\right]$ and attention selective $\left[\mathrm{F}_{(1,64)}=14.531, p=.001\right]$. Thus, it was observed that the experimental group improved not only its motor performance, but also significantly improved the performance of cognitive functions tested.

Keywords: executive function, child development, school physical education

Submetido: 25.06.2012 | Aceite: 15.05.2013

Cintia Mota Cardeal. Universidade Federal do Recôncavo da Bahia, Brasil.

Lílian Alves Pereira, Pollyanna Ferreira da Silva, Nanci Maria de França. Universidade Católica de Brasília, Brasil. Endereço para correspondência: Cintia Mota Cardeal, Centro de Formação de Professores/UFRB, Av. Nestor de Melo Pita, n. 535, Centro, Amargosa/BA, CEP: 45.300-000, Brasil.

E-mail: cintiacardeal@gmail.com 
Aliado a maturação biológica o desenvolvimento das capacidades motoras garante ao indivíduo condições de movimentar-se de maneira contínua, em ritmos variados e de diversas formas. Entretanto, há que se considerar que as alterações ocorridas no sistema motor da criança ocorrem concomitantemente ao desenvolvimento do sistema cognitivo e, portanto, não há como dissociar esses dois aspectos do desenvolvimento, pois ambos coexistem no mesmo organismo. Nessa tentativa de observar relação entre o sistema cognitivo e a atividade física, alguns pesquisadores observaram benefícios adquiridos pela cognição advinda das atividades físicas em crianças com idade escolar (Tomporowski, 2003; Hillman, Castelli, \& Buck 2005).

Ao relacionar exercícios físicos e função executiva verificou-se uma melhora significativa na função executiva de crianças em idade escolar (Ahamed et al., 2007). Além disso, Budde et al. (2008) mostraram que a atenção e a concentração dos escolares melhoraram após a prática de exercícios coordenativos. Assim como, a atividade física crônica melhorou o desempenho em tarefas atencionais, em pré-escolares, como ficou demonstrado nos estudos de (Castelli, Hillman, Buck, \& Erwin, 2007; Hillman et al., 2009).

Dentre os elementos que compõe a cognição está a função executiva, cuja qual é composta pelo sistema atencional, memória de trabalho, raciocínio e flexibilidade. Tanto a atenção quanto à memória compõem uma rede que se estabelece no momento da aprendizagem, portanto são pontos importantes a serem considerados quando há o intuito de melhorar o nível de aprendizagem do aluno. A atenção seletiva pode ser definida como: a capacidade de focalizar recursos mentais sobre as informações que são mais relevantes em um dado momento, ou seja, é a capacidade "filtrar" uma grande quantidade de estímulos sensoriais irrelevantes, a fim de concentrar naqueles de maior importância (Sternberg, 2000).

Já a memória pode ser dividida quanto à duração: 1-memória sensorial, cuja retenção não dura mais que alguns segundos; 2-memória de curto prazo (ou memória de trabalho), que dura minutos ou horas e que proporciona continuidade e sentido ao presente; 3-memória de longo prazo, que dura dias, semanas ou anos estabelecendo traços duradouros no sistema cognitivo (Sternberg, 2000). Nessa perspectiva temporal, a memória de trabalho está associada intimamente com o processo de focalização da atenção e seleção da informação, visto que, a memória de trabalho é um sistema ativo no processamento dessas informações e não funciona apenas como um reservatório de informações temporárias (Baddeley, 2000). Assim, o termo "memória de trabalho" é utilizado para se referir a um sistema de multicomponentes, capaz de armazenar e manipular informações desempenhando um papel importante na complexa atividade cognitiva, como: a aprendizagem, a compreensão e a argumentação (Gathercole, 1998).

Mesmo considerando a importância da memória de trabalho e da atenção seletiva no processo de aprendizagem quer seja motora, quer seja de elementos teórico-abstratos, faz-se necessário considerar o processo de desenvolvimento e maturação, visto que, tanto a infância quanto a adolescência são marcadas por um período dinâmico nas diferentes dimensões do desenvolvimento (ex.: cognitivo, motor, emocional e habilidades perceptivas) (Barnea-Goraly et al., 2005). Esse desenvolvimento está associado ao volume do córtex, especialmente ao córtex frontal (Casey, Giedd, \& Thomas, 2000). Neste sentido, o lobo frontal funciona como uma "central" para a cognição humana; que assimila, executa e organiza os processos cognitivos; realiza os processos emocionais e transfere informações para as outras áreas cerebrais. Neste contexto a maturação do córtex pré-frontal é a base para o aumento na eficiência do controle executivo que torna a aprendizagem mais fácil.

Desse modo, um conjunto crescente de 
estudos tem evidenciado que o desenvolvimento motor e o exercício físico podem apresentar uma correlação positiva com a cognição, como observado por Rigoli, Piek, Kane \& Oosterlaan (2012) ao associar a coordenação motora, desempenho acadêmico mediado pela memória de trabalho, em uma amostra composta por 93 adolescentes. Em relação às alterações estruturais do sistema nervoso quando associado à atividade física, verifica-se que pode ocorrer um aumento do hipocampo, aumento no volume do glânglio basal, bem como ocorre a melhora da memória, atenção e flexibilidade cognitiva (Chaddock et al., 2012; Voss et al., 2011; Pontifex et al., 2010), na constatação dos resultados dessas pesquisas, nota-se que o pico do desenvolvimento cognitivo ocorre entre as idades de 5 e 15 anos, sendo que esse é o período crucial para o desempenho acadêmico. Além disso, esses achados têm implicações significantes tanto para a educação quanto para a saúde pública.

Portanto, a proposta desse estudo é contribuir na discussão sobre a prática da Educação Física Escolar, seus benefícios e importância no contexto da formação do indivíduo. Visa também despertar para necessidade de pesquisar a motricidade e sua relação com o desenvolvimento da cognição, pois apesar de haver estudos que relacionam atividade física e cognição, não há um consenso entre os resultados e também há poucos estudos que direcionam especificamente a relação entre educação física escolar, desenvolvimento motor e processos cognitivos. Desse modo, o presente estudo teve como objetivo verificar o efeito da estimulação motora, nas respostas da função cognitiva de crianças na faixa etária de 6 a 10 anos, de escolas públicas do Distrito Federal, Brasil.

\section{MÉTODO}

\section{Amostra}

Este estudo foi realizado com alunos do Bloco Infantil de Alfabetização (BIA) 2 e 3 do ensino fundamental, com idade entre 6 a 10 anos de ambos os sexos, de 2 escolas públicas localizadas na cidade de Taguatinga, no Distrito Federal. A escolha das escolas se deu de forma intencional, pois os gestores, não fizeram objeção ao cronograma e nem a proposta do estudo. Todas as turmas participaram do experimento assim, foram estabelecidos dois grupos sendo um grupo experimental subdividido em três turmas distintas (GE, $n=40$ ) e um grupo controle (GC, $n=40$ ). O grupo controle não participou da intervenção, enquanto o grupo experimental realizou aulas de educação física escolar, duas vezes na semana com duração de 50min, durante 7 meses. Todos os sujeitos foram testados antes e após o experimento nas variáveis analisadas por este estudo.

\section{Procedimentos Éticos}

A pesquisa foi aprovada pelo Comitê de Ética da Universidade Católica de Brasília, parecer: CEP/UCB No130/2005. Ocorreram dois encontros com todos os pais e responsáveis, o primeiro para apresentar os objetivos da pesquisa, bem como, os procedimentos e instrumentos utilizados para que todos fossem esclarecidos. Todos os responsáveis legais das crianças participantes da pesquisa assinaram o Termo de Consentimento Livre e Esclarecido conforme diretrizes do CNS 196/96 para pesquisa com seres humanos no Brasil. O segundo encontro ocorreu para que fossem apresentados os resultados da pesquisa de forma individual para cada criança participante.

\section{Critérios de Inclusão}

Participaram da intervenção todos os alunos regularmente matriculados no BIA, porém, para ser incluída na analise final do estudo a criança deveria ter participado de todas as aulas, bem como, das avaliações no pré-intervenção e pós-teste; estar entre os estágios 1 e 2 da escala de Tanner (1962) para maturação sexual e não apresentar disfunções mentais ou psicológicas e nem problemas que impedisse sua locomoção. Ao total 6 alunos foram excluídos dessa 
análise por serem diagnosticados portadores de hiperatividade (por psicólogos do Núcleo de Apoio Psicológico da Secretaria de Educação do Distrito Federal), entretanto todos participaram das aulas de Educação Física escolar.

\section{Instrumentos}

Avaliação Antropométrica: Os dois grupos foram mensurados nas variáveis antropométricas para a caracterização da amostra, quanto ao peso, estatura e índice de massa corporal (IMC). Os instrumentos utilizados foram: Estadiômetro da marca Seca, (modelo 206) com $200 \mathrm{~cm}$ de comprimento e resolução de $1 \mathrm{~mm}$ fixado em uma parede plana. Balança digital modelo Plena precisão de $100 \mathrm{~kg}$ e resolução de $1 \mathrm{~kg}$. Fita de métrica Seca com $200 \mathrm{~cm}$ de comprimento e resolução de $1 \mathrm{~mm}$.

Avaliação Motora: Os testes motores avaliaram: coordenação motora fina, coordenação motora ampla, equilíbrio, esquema corporal, lateralidade, organização temporal e organização espacial. Os testes foram validados por Rosa Neto, (2001) para estimar a idade motora de cada elemento avaliado. Todos os alunos começaram com provas motoras equivalentes há dois anos inferiores à sua idade cronológica, e se o aluno não conseguisse completar o primeiro teste aplicar-se-ia o teste da idade anterior. O tempo gasto para a aplicação dos testes com cada criança foi de aproximadamente 40 minutos. O material utilizado para a realização dos testes está incluso no "kit" de Avaliação Motora -EDM (Rosa Neto, 2001). Os dados coletados resultam na idade motora da criança expressa em meses.

Teste de Atenção Seletiva (Stroop): Este teste avaliou a atenção seletiva e só pode ser aplicado em crianças já alfabetizadas. O teste foi realizado individualmente, com duração de $5 \mathrm{~min}$. O software do Stroop foi adaptado à realidade brasileira por Córdova, Karnikowski, Pandossio e Nóbrega (2008). O teste consiste em três etapas: na primeira fase aparece em destaque na tela do computador um retângulo pintado de uma cor, e os nomes de duas cores escritas abaixo. Esses nomes de cores são escritos com a mesma cor do nome indicado. Desse modo, a criança olha a cor do retângulo e associava com o nome da cor escrita logo abaixo, para responder a criança aperta as setas < ou $>$. São apresentados 13 estímulos em cada fase, e as cores apresentadas em todo o teste são: verde, vermelho, azul e preto.

A segunda fase começa sem que haja qualquer parada, ou seja, as três fases são contínuas. Nessa fase as palavras estão escritas em branco, aparece em destaque o nome de uma cor. A criança responde então, com as setas onde está escrito o mesmo nome correspondente. Já na última fase, a palavra em destaque está escrita com cor diferente em relação ao nome. A criança passa desse modo, a não responder o nome que está escrito, mas sim, a cor que está pintada à palavra.

Teste de Reação Simples (TEVA): Este teste foi aplicado tanto em crianças não alfabetizadas como nas alfabetizadas, tem por objetivo avaliar o estado de alerta primário, sendo responsável pela a primeira reação do indivíduo a um estímulo, bem como a seleção de estímulo (Córdova, 2005).

$\mathrm{O}$ teste se inicia com um quadrado pequeno piscando continuamente no centro da tela do computador, a criança é orientada a apertar o mais rápido possível a tecla espaço todas as vezes que um quadrado grande aparecer na tela. Da mesma forma que o teste de Stroop, o TEVA é analisado através do tempo de resposta da criança ao estímulo oferecido.

As configurações em relação aos estímulos são programadas, o tempo total do teste é de 3 min com 180 estímulos. A duração do estímulo na tela foi de $700 \mathrm{~ms}$ com um intervalo de $300 \mathrm{~ms}$. O teste foi dividido em 3 blocos, cada bloco com 1 minuto cada. O alvo (quadrado maior) aparecia de forma aleatória, distribuído em 20 aparições em 3 min no total.

Teste de Função Executiva (Torre de Hanói): Objetiva a mensuração da função executiva 
(Bull, Espy, \& Senn, 2004). A Torre de Hanói se constitui em três pinos com seis discos, porém nessa pesquisa foram utilizados três discos. $\mathrm{O}$ objetivo está em transportar a torre inicial para qualquer outro pino vazio, podendo a criança deslocar um disco de cada vez, não sobrepor um disco maior sobre um disco menor e nem segurar os discos nas mãos enquanto move outros. A criança foi orientada para que tentasse solucionar o problema o mais rápido possível, já que o tempo seria contado.

\section{Procedimentos}

Intervenção motora: A intervenção contou com duas aulas semanais. Todas as aulas foram planejadas de acordo com as capacidades perceptivomotoras, por meio de jogos e exercícios como já foi sugerido por (Le Boulch, 1988). Um cronograma foi estabelecido para dispor de forma igual cada um dos elementos perceptivo motores tanto na condição de habilidade básica quanto na condição de habilidade específica, de tal maneira que todos fossem contemplados com a mesma quantidade de horas/aulas. Nos planos de aula elaborados constavam todas as atividades sequencialmente descritas, bem como, o modo de sua aplicação, os materiais necessários e os objetivos a serem atingidos tanto para o professor quanto para o aluno. As atividades tinham duração de $50 \mathrm{~min}$, e as três turmas realizavam as aulas separadamente.

Em todas as aulas foram observados: o comportamento, as dificuldades, as superações e o interesse apresentado pelas crianças. Essas observações foram transcritos em relatórios para posteriormente avaliar qualitativamente o progresso da criança durante o período do experimento e relatá-los aos pais.

A escola não oferecia aulas regulares de Educação Física para os alunos, por isso tinha poucos materiais disponíveis para a prática. Desse modo, durante o período da intervenção, as aulas foram elaboradas com a finalidade de aproveitar o pouco material que havia na escola e alguns materiais alternativos como: jornal e sucatas. Para a construção das atividades foram utilizados; cordas, arcos, bolas, colchonetes, cones, tintas, papéis, balões, jornais, fitas, elásticos, lenços, garrafas pet, latas, giz e panos de TNT. Vale a pena ressaltar que, no Distrito Federal, as escolas públicas de ensino fundamental não contam com professor especialista de educação física, ficando esta função a cargo do professor da sala. Por isso todos os materiais, planos de ensino, bem como as técnicas para desenvolver material pedagógico alternativo foram doados para as escolas para que os professores pudessem desenvolver as atividades com as crianças após o termino do experimento.

\section{Análise Estatística}

Essa foi uma pesquisa de caráter experimental. Realizou-se inicialmente a análise exploratória dos dados com a finalidade de avaliar os casos faltosos, os parâmetros de normalidade, linearidade e casos a serem excluídos (outsiders). Foram realizadas comparações intra-grupo (pré e pós interferência) e entre os grupos nos dois momentos (pré e pós), para cada uma das variáveis dependentes separadamente. Foi realizada Análise de Variância Anova spli-plot (2x2), sendo as variáveis independentes: grupos (experimental e controle) e tratamento pré e pós-intervenção, já as variáveis dependentes foram as variáveis psicomotoras (motricidade fina, motricidade ampla, equilíbrio, esquema corporal, organização temporal e organização espacial) e as cognitivas (Função executiva e atenção). Também foi utilizado para a análise de variância dos grupos o teste Wilki's Lambda, para avaliar as interações e diferenças ocorridas em ambos os grupos após o período de tratamento.

Uma análise suplementar foi realizada para o subgrupo formado, com as crianças que realizaram o teste de atenção seletiva, por estarem alfabetizadas. Desse modo, as mesmas análises empregadas para as outras variáveis foram realizadas em amostra composto em 36 indivíduos do GE e 30 do GC. Os testes Post hoc de Sheffé e Dunneth'C foram aplicados para comparações 
múltiplas para uma significância de $p<.05$.

\section{RESULTADOS}

Todas as crianças foram avaliadas quanto às características antropométricas, a fim de caracterizar o padrão antropométrico de ambos os grupos (tabela 1). Quanto ao estágio maturacional $75 \%$ do total de crianças se encontraram no primeiro estágio e $35 \%$ no estágio 2 segundo a escala de Tanner (1962).

Os resultados da avaliação motora realizada antes da intervenção, descritos na tabela 2 , mostraram que as variáveis motoras mais defasadas foram: organização temporal, organização espacial e esquema corporal em ambos os grupos.

Após a intervenção observou-se que tanto para grupo experimental quanto para grupo controle, houve uma melhora na motricidade. Esperava-se que essas melhoras ocorressem, visto que, esses alunos estão em franco processo de desenvolvimento e maturação, entretanto ressalta-se uma melhora substancial do grupo experimental quando comparado com o pré-teste. Desse modo, resultados pré e pós-testes das variáveis motoras foram avaliadas através do modelo estatístico
ANOVA (2x2). A avaliação inferencial revela que houve uma interação significativa entre fator grupo e cada uma das variáveis motoras analisadas, houve também um contraste significativo entre dois momentos de avaliação $\left[\mathrm{F}_{(1,118)}=70.569 ; p=.001\right]$.

Quanto a observação das mudanças ocorridas entre grupos mostrados na tabela 3, constatou-se que as mudanças ocorridas no grupo experimental podem ser atribuídas ao tratamento realizado, uma vez que apresentou melhora significativa. As crianças partiram de déficit motor de 14.01 meses, em média, para uma idade positiva de 6.19 meses, enquanto o grupo controle apresentou uma variação de dois meses na idade motora, porém mantendo-as em déficit motor de 15.69 meses em média.

Do mesmo modo, foi realizada a análise descritiva das variáveis cognitivas de ambos os grupos. As avaliações do teste de Função Executiva (Torre de Hanói) e o teste de atenção (TEVA), os resultados estão descritos na tabela 4.

Nas avaliações pós-interferência observou-se uma redução no tempo de realização do teste em ambos os grupos. Diminuiu também o número de movimentações caindo de 13.36 para 8.66 em média no grupo experimental.

Tabela 1

Média e Desvio Padrão das Variáveis Antropométricas dos Grupos controle e experimental subdivididos por sexo

\begin{tabular}{cccccc}
\hline \multirow{2}{*}{ Variáveis } & \multicolumn{2}{c}{ Grupo Controle $(n=40)$} & & \multicolumn{2}{c}{ Grupo Experimental $(n=40)$} \\
\cline { 2 - 3 } \cline { 5 - 6 } & Feminino & Masculino & & Feminino & Masculino \\
\hline Peso $(\mathrm{Kg})$ & $25.435(4.3)$ & $26.748(1.270)$ & & $26.296(4.494)$ & $27.094(5.681)$ \\
Estatura $(\mathrm{m})$ & $1.285(0.049)$ & $1.270(0.049)$ & & $1.268(0.069)$ & $1.277(0.065)$ \\
$\mathrm{IMC}\left(\mathrm{Kg} / \mathrm{m}^{2}\right)$ & $15.351(4.949)$ & $16.523(1.758)$ & & $16.243(2.369)$ & $15.276(3.324)$ \\
\hline
\end{tabular}

Tabela 2

Média e Desvio Padrão da Avaliação Motora Pré e Pós-Intervenção

\begin{tabular}{cccccc}
\hline \multirow{2}{*}{ Variáveis } & \multicolumn{2}{c}{ Antes da Intervenção } & & \multicolumn{2}{c}{ Após a Intervenção } \\
\cline { 2 - 3 } \cline { 5 - 6 } & ${ }^{*} \mathrm{GE}$ & ${ }^{* *} \mathrm{GC}$ & & ${ }^{*} \mathrm{GE}$ & ${ }^{* *} \mathrm{GC}$ \\
\hline Motricidade Fina & $90.20(11.26)$ & $84.53(11.65)$ & & $104.60(12.66)$ & $86.10(8.89)$ \\
Motricidade Ampla & $89.53(12.88)$ & $92.80(13.18)$ & & $111.50(16.54)$ & $95.80(13.02)$ \\
Equilíbrio & $84.80(19.65)$ & $84.53(17.26)$ & & $112.40(20.25)$ & $87.87(18.08)$ \\
Esquema Corporal & $83.40(17.31)$ & $79.90(12.76)$ & & $98.40(18.59)$ & $82.00(13.37)$ \\
Organização Espacial & $69.50(15.43)$ & $74.60(9.77)$ & & $98.62(17.27)$ & $77.30(9.82)$ \\
Organização Temporal & $67.60(9.87)$ & $65.80(6.43)$ & & $77.80(6.80)$ & $69.00(6.10)$ \\
Idade Motora Geral & $80.91(9.27)$ & $80.48(7.37)$ & & $105.69(10.16)$ & $84.97(7.48)$ \\
Razão Motora & $-14.01(10.13)$ & $-18.09(7.62)$ & & $6.19(9.38)$ & $-15.69(7.71)$ \\
\hline
\end{tabular}

${ }^{*} \mathrm{GE}=$ Grupo Experimental; ${ }^{*} \mathrm{GC}=$ Grupo Controle. A média e o desvio padrão das variáveis analisadas estão expressas em meses. 
Enquanto no grupo controle a primeira avaliação apresentou uma média de 14.140 movimentações e na segunda 12.05 .

O teste de Stroop foi analisado separadamente, pois alguns alunos não estavam alfabetizados. Na tabela 5 estão descritos as médias e os desvios padrão para os dois momentos da pesquisa.

Para cada fase do teste foi calculado a média do tempo de reação motora (em segundos), ou seja, os valores descritos na tabela são referentes ao tempo médio de resposta ao estímulo em cada fase. Tanto no grupo controle quanto no grupo experimental observamos as mesmas características. Em ambos os momentos houve o aumento no tempo de resposta a cada fase do teste.

$\mathrm{Na}$ avaliação referente à função executiva, testada pelo teste da Torre de Hanói (tabela 6), verifica-se contraste significativo dentro de cada grupo, bem como uma diferença significativa entre os grupos após o período de estimulação motora $\left[\mathrm{F}_{(1,118)}=13.768 ; p=.001\right]$, observa-se também que o grupo experimental além de obter uma melhora no tempo execução do teste, também errou menos. O mesmo ocorreu com o teste de reação simples TEVA, o qual apresentou uma diferença significativa entre os grupos $\left[\mathrm{F}_{(1,118)}=18.352 ; p=.001\right]$. O grupo que sofreu tratamento apresentou resultados significativamente melhores quando comparados com o grupo controle, ou seja, a média de tempo para a resolução do teste de reação caiu de 131.5 segundos para 57.16 segundos no grupo experimental, já no grupo controle diminuiu 144.17 para 125.49 segundos. Os resul-

Tabela 3

Análise do Efeito dentro e entre os Grupos Controle e Experimental nas variáveis motoras

\begin{tabular}{|c|c|c|c|c|c|c|c|c|}
\hline \multirow{2}{*}{ Variáveis } & \multicolumn{4}{|c|}{ Efeito da Intervenção intra Grupos } & \multicolumn{4}{|c|}{ Efeito da Intervenção entre os Grupos } \\
\hline & $\mathrm{df}$ & $\mathrm{F}$ & sig. & Observard Power a & $\mathrm{df}$ & $\mathrm{F}$ & sig. & Observard Power a \\
\hline Motricidade Fina & 1 & 45.608 & 0.001 & 1.000 & 1 & 12.780 & 0.001 & 0.997 \\
\hline Motricidade Global & 1 & 50.566 & 0.001 & 1.000 & 1 & 8.140 & 0.001 & 1.000 \\
\hline Equilíbrio & 1 & 76.159 & 0.001 & 1.000 & 1 & 15.516 & 0.001 & 1.000 \\
\hline Esquema Corporal & 1 & 28.980 & 0.001 & 1.000 & 1 & 14.576 & 0.001 & 1.000 \\
\hline Organização Espacial & 1 & 91.141 & 0.001 & 1.000 & 1 & 14.533 & 0.001 & 0.964 \\
\hline Organização Temporal & 1 & 19.624 & 0.001 & 1.000 & 1 & 9.329 & 0.001 & 0.853 \\
\hline Idade Motora Geral & 1 & 109.100 & 0.001 & 1.000 & 1 & 8.543 & 0.001 & 0.821 \\
\hline Razão da Idade Motora & 1 & 237.612 & 0.001 & 1.000 & 1 & 4.293 & 0.001 & 0.532 \\
\hline
\end{tabular}

a alfa $=.05$

Tabela 4

Média e Desvio Padrão dos Testes Cognitivos Pré e Pós-Intervenção

\begin{tabular}{cccccc}
\hline \multirow{2}{*}{ Variáveis } & \multicolumn{2}{c}{ Antes da Intervenção } & & \multicolumn{2}{c}{ Após a Intervenção } \\
\cline { 2 - 3 } \cline { 5 - 6 } & \multicolumn{6}{c}{$\mathrm{GE}^{*}$} & GC & & GE & GC* \\
\hline Hanói (seg.) & $131.59(69.11)$ & $144.17(5.23)$ & & $57.16(21.41)$ & $125.49(79.71)$ \\
Movimentações & $13.36(4.68)$ & $14.40(5.67)$ & & $8.66(2.97)$ & $12.05(4.59)$ \\
Erros - Hanoi & $2.27(1.75)$ & $2.72(1.28)$ & & $1.34(0.58)$ & $2.53(1.56)$ \\
TEVA (seg.) & $0.64(0.07)$ & $0.62(0.07)$ & & $0.58(0.07)$ & $0.604(0.079)$ \\
Erros - TEVA & $2.88(0.73)$ & $3.08(0.53)$ & & $1.7(0.7)$ & $2.68(1.79)$ \\
\hline
\end{tabular}

${ }^{*} \mathrm{GE}=$ Grupo Experimental; ${ }^{* *} \mathrm{GC}=$ Grupo Controle; todos os grupos foram testados para uma amostra de 60 crianças antes e depois da intervenção.

Tabela 5

Média e Desvio Padrão do Teste de Stroop Pré e Pós-Intervenção

\begin{tabular}{cccccc}
\hline \multirow{2}{*}{ Variáveis } & \multicolumn{2}{c}{ Antes da Intervenção } & & \multicolumn{2}{c}{ Após Intervenção } \\
\cline { 2 - 3 } \cline { 5 - 6 } & $*$ GE $(n=36)$ & ${ }^{* *} \mathrm{GC}(n=30)$ & & $*$ GE $(n=36)$ & ${ }^{* *} \mathrm{GC}(n=30)$ \\
\hline Stroop 1 & $2.563(0.64)$ & $2.429(0.91)$ & & $2.249(0.49)$ & $2.478(0.53)$ \\
Stroop 2 & $2.960(0.69)$ & $3.153(0.59)$ & & $2.666(0.51)$ & $2.815(0.74)$ \\
Stroop 3 & $3.474(0.97)$ & $3.889(0.62)$ & & $3.158(0.62)$ & $3.576(0.59)$ \\
Erros Stroop & $4.80(2.11)$ & $7.06(2.45)$ & & $2.91(0.97)$ & $6.36(3.18)$ \\
\hline
\end{tabular}


Tabela 6

Análise do Efeito do Treinamento na memoria e atenção entre os Grupos

\begin{tabular}{ccccc}
\hline Variáveis & df & F & sig. & Observard Powera \\
\hline Hanói & 1 & 12.780 & 0.001 & 0.997 \\
Erros de Execução - Hanói & 1 & 8.140 & 0.001 & 1.000 \\
TEVA & 1 & 15.516 & 0.001 & 1.000 \\
Erros de Execução - TEVA & 1 & 14.576 & 0.001 & 1.000 \\
Stroop 1 & 1 & 14.533 & 0.001 & 0.964 \\
Stroop 2 & 1 & 9.329 & 0.001 & 0.853 \\
Stroop 3 & 1 & 8.543 & 0.001 & 0.821 \\
Erro de Execução - Stroop & 1 & 4.293 & 0.001 & 0.532 \\
\hline
\end{tabular}

tados da análise no tempo de reação mostram uma diferença significativa dentro dos grupos $\left[\mathrm{F}_{(1,118)}=61.315 ; p=.001\right]$.

Quando se empregou a mesma avaliação inferencial para a variável atenção seletiva (Stroop), observamos que ocorreu interação significativa dentro dos grupos $\left[\mathrm{F}_{(7,58)}=5.206\right.$; $p=.001]$, do mesmo modo houve diferença significativa entre os grupos $\left[\mathrm{F}_{(1,64)}=14.531\right.$; $p=.001]$. Finalmente, por esses resultados pode-se inferir que houve uma resposta positiva ao tratamento oferecido para as crianças do grupo experimental tanto para variáveis motoras quanto para variáveis cognitivas.

\section{DISCUSSÃO}

O desenvolvimento das habilidades perceptivo-motoras na criança ocorre em diversos ritmos e influenciam-se mutualmente. A partir dos resultados obtidos na avaliação motora observa-se que o esquema corporal, a organização espacial e a organização temporal foram as que apresentaram o maior grau de defasagem para ambos os grupos. O processo de desenvolvimento do esquema corporal ocorre plenamente durante a infância, no qual, a criança toma consciência das partes que constituem o corpo e como essas podem se movimentar. $\mathrm{Na}$ sua fase final (entre 7 e 8 anos de idade), está intimamente associada com o desenvolvimento espacial.

Porém, a organização espacial depende também do desenvolvimento da acuidade visual, o que nas idades aqui observadas estão em fase de desenvolvimento mais lento e não chegaram a sua maturidade. Os conceitos de localização subjetiva e auto-espaço estão atrelados à fase do pensamento pré-operatório. Já o conceito de localização objeto-espaço está conectado às estruturas cognitivas superiores, a exemplo da memoria espacial atrelada ao aumento do hipocampo, na fase de operações concretas (Piaget, 1978; Gallahue \& Ozmun, 2005).

A organização temporal, como foi descrita por Piaget (1978), é a mais complexa, portanto desenvolve-se mais lentamente. Quando juntas, a organização espaço-temporal decorre da integração cortical das estruturas visuais (lobo occipital) e temporais (lobo temporal). Nessa fase as crianças estão em desenvolvimento, sendo este o período em que ocorre o pico de desenvolvimento e maturação dessas estruturas (Pfferbaum et al., 1994; Erickson et al., 2011). Portanto, apesar de serem estruturas que se desenvolvem mais tardiamente, nesses alunos avaliados existia um déficit motor importante, visto que, essas organizações influenciam na aprendizagem da leitura e escrita bem como nas operações da aritmética. Do ponto de vista pedagógico é o momento ideal para assimilar os estímulos do ambiente. Indicando que um ambiente de aprendizagem, rico, multissensorial em conexão com o mundo real é essencial para o pleno desenvolvimento da criança.

$O$ déficit motor das crianças analisadas, neste estudo, estava acima da média quando comparados com outros estudos que utilizaram os mesmos métodos de análise. Como no estudo realizado por Souza Neto, Micotti, Benites, Silveira, e Alves (2005) em 23 crianças com idades entre 9 e 10 anos de uma escola 
pública de Rio Claro, $60 \%$ delas estavam com déficit motor. Em outra pesquisa realizada por Pellegrini et al. (2003) também em escola pública, observou-se que as crianças que frequentavam o primeiro, segundo e terceiro ano de alfabetização ( $n=77$ alunos) também eram acometidas de déficit motor, a sua maioria estava abaixo da categoria normal médio, cerca de $58 \%$ da amostra.

Segundo Pfferbaum, Mathalon, Rawles, Zipursky, e Lim (1994), Romine e Reynolds (2004), as estruturas morfo-cerebrais, como as estruturas corticais superiores e o processo de mielinização estão, na segunda infância, em desenvolvimento acelerado. Da mesma forma, Rosa Neto (2001) e Gallahue e Ozmun (2005) relatam que é também na segunda infância que os sistemas motores estão em desenvolvimento, porém alguns sistemas já começam a diminuir o processo de desenvolvimento e o organismo parte para a obtenção do refinamento da habilidade.

Através da comparação entre pré e pós-testes, pode-se concluir que ocorreram melhoras significativas tanto das variáveis motoras quanto das variáveis cognitivas. $\mathrm{O}$ tempo de intervenção foi um fator importante, pois, Souza Neto et al. (2005) realizaram pesquisa com estimulação motora avaliando alunos com idades entre 8 e 10 anos, utilizando o protocolo elaborado por Rosa Neto (2001), com intervenção de 3 meses. Os resultados por eles obtidos não foram estatisticamente significativos nesse período. Como a proposta do presente estudo foi de um período maior de intervenção pode-se inferir que, em ambos os grupos houve uma melhora nas variáveis motoras testadas, mesmo sendo diagnosticado déficit motor acentuado no pré-teste.

O desenvolvimento motor na infância segundo Santos, Dantas, e Oliveira (2004), caracteriza-se pela aquisição de habilidades motoras, que possibilitaram à criança desenvolver o domínio do seu corpo em diferentes posturas, locomoções e manipulações. Dessa forma essas habilidades básicas formam o alicerce para que essas crianças possam desenvolver suas tarefas diárias. Em outro estudo com a mesma população, porém, com amostra diferente daquela avaliada no presente artigo, Senra (2007) evidenciou melhoras significativas, nas habilidades funcionais de autocuidado, de mobilidade e função social, avaliadas pelo instrumento PEDI, após um programa de estimulação semelhante, mas adaptada para o meio aquático.

Em estudo mais recente, Amaro, Gomes, Santos, e Rosa Neto (2010) observaram que crianças com dificuldades de aprendizagem também apresentavam déficit motor. Da mesma forma, Medina-Papst e Marques (2010) encontraram a mesma associação entre a dificuldade de aprendizagem e déficit motor. Ambos os estudos utilizaram os mesmos instrumentos de avaliação motora o EDM. Demonstrando assim, que o atraso no desenvolvimento motor pode estar associado a outros problemas ocorridos concomitantemente ao desenvolvimento do indivíduo. Outro estudo utilizando o protocolo EDM verificou que há também uma associação entre o desenvolvimento da coordenação motora com a atenção em crianças de 7 a 11 anos, no qual foi verificado que as crianças que tinham Transtorno de Déficit Atencional e Hiperatividade (TDH) do tipo combinado apresentaram atrasos na coordenação motora em relação às crianças que não foram diagnosticadas com TDH (Goulardins et al. 2013).

Desse modo, o exercício físico melhora a saúde mental em jovens e adultos (Colcombe \& Kramer, 2003, Tomporowski, 2003, Middleton et al., 2011). Porém, quando se trata do desenvolvimento infantil existem poucos estudos mostrando a influência da atividade física na cognição. Num dos poucos estudos, Wassenberg et al. (2005), relacionaram o desempenho cognitivo com o desempenho motor em 400 crianças de cinco e seis anos, e não encontraram correlação consistente entre esses dois fatores. É importante destacar que nessa pesquisa, o desenho experimental estabeleceu o desempenho 
motor como variável dependente e o desempenho cognitivo como variável independente, ou seja, os autores tentaram avaliar a influência da cognição sobre os processos motores.

O presente estudo encontrou diferenças significativas entre dois grupos no que se refere ao tempo de reação, que pode estar associado com a atividade física, corroborando com (Chaddock et al., 2012), do mesmo modo com processo de inibição (Georgopoulos, 2000). Para Ladewig (2000), a atenção seletiva, no qual o processo de inibição de estímulo está presente, determina o que é percebido e codificado na memória que por sua vez, poderá facilitar a recuperação da informação. Portanto, fazendo um paralelo quando a criança entra no ensino fundamental, os processos atencionais estão sofrendo mudanças e atingem um estágio no qual a criança é facilmente distraída por inúmeras informações contidas no ambiente, ou seja, elas ainda não são capazes de separar as informações relevantes das irrelevantes, apresentando dificuldades na focalização da atenção.

Quanto à função executiva foi observado, que o grupo experimental obteve melhores respostas no momento pós-intervenção motora quando comparado com o grupo controle. O que corrobora com as afirmações de Diamond (2000), que a execução de movimentos motores complexos também recruta circuito neural associados com a função executiva. Adicionalmente, a função executiva é fundamental para todas as formas de comportamento e é uma estrutura fundamental para o desenvolvimento. É importante para o comportamento em sala de aula, para a aprendizagem e também para a auto-regulação. Assim a atividade física, aqui representada pela educação física escolar, tem o potencial de promover o desenvolvimento através do seu impacto direto na função executiva (Blair \& Diamond, 2008).
Culturalmente os objetos de estudo "corpo" e "mente" tem sido tratados de maneira cartesiana, a partir de uma visão racionalista que vários campos da ciência utilizam para a obtenção dos resultados de pesquisa. No entanto, o ser humano é constituído de partes que no todo formam um ser que pensa que se move e interage. Esse estudo buscou testar a hipótese da relação entre o desenvolvimento da motricidade e da cognição na criança, mesmo considerando as limitações do experimento devido à variáveis intervenientes como os estímulos recebidos no tempo livre fora do ambiente escolar, tipo de alimentação. Mesmo assim, pontuamos a importância da educação física no ensino fundamental como meio efetivo de auxílio para as aprendizagens motoras e cognitivas. A educação física com caráter participativo, no contexto escolar, com aulas elaboradas e com objetivos bem delineados, procurando atender as características de cada fase do desenvolvimento que esses alunos se encontram, realmente faz-se necessária. Acreditamos que este trabalho é mais um argumento na direção do reconhecimento social da "significação do movimento" para o desenvolvimento da criança.

Foi observado que, o grupo que sofreu intervenção melhorou não somente o aspecto motor, mas também melhorou de forma significativa o desempenho nos testes de função executiva e atenção seletiva, ou seja, o raciocínio ainda que baseado nas operações concretas tornou-se mais rápido requisitando menos tempo para solucionar um problema.

\section{Agradecimentos:}

Às instituições de fomento à pesquisa CAPES e CNPq.

Conflito de Interesses:

Nada declarado.

Financiamento:

Nada declarado. 
Ahamed, Y., MacDonald H., Reed K., Naylor P. J., Liu-Ambrose T., \& McKay H. (2007). School-based physical activity does not compromise children's academic performance. Medicine and Science in Sports and Exercise, 39(2), 371-376. doi: 10.1249/01.mss.0000241654.45500.8e

Amaro, K. N.; Gomes, L. J., Santos, A. P. M., Rosa Neto, F. (2010). Desenvolvimento motor em escolares com dificuldades na aprendizagem. Movimento \& Percepção (Online), (11), 35-42,

Baddeley, A. D. (2000). The episodic buffer: a new compenent in working memory? Trends in Cognitive Sciences, 4(11), 417-423. doi:10.1016/ S1364-6613(00)01538-2

Barnea-Goraly, N., Menon, V., Eckert, M., Tamm, L., Bammer, R., Karchemskiy, A., \& Reiss, A.L. (2005). White Matter Development During Childhood and Adolescence: a cross-sectional diffusion tensor imaging study. Cerebral Cortex, 15(12), 1848-1854. doi: 10.1093/cercor/bhi062

Blair, C., \& Diamond, A. (2008). Biological processes in prevention and intervention: the promotion of self-regulation as a means of preventing school failure. Development Psychopathology, 20(3), 899-911. doi: 10.1017/ S0954579408000436

Budde, H., Voelcker-Rehage, C., Pietraßyk,Y., K-Kendziorra, S., Ribeiro, P., \& Tidow, G. (2008). Acute coordinative exercise improves attentional performance in adolescents. Neurosci Lett, 441 (2), 219-223. doi: 10.1016/j.neulet.2008.06.024

Bull, R., Espy, K. A., \& Senn, T. E. (2004). A Comparison of Performance on the Towers of London and Hanoi in Young Children. Journal of Child Psychology and Psychiatry, 45(4), 743-754. doi: 10.1111/j.1469-7610.2004.00268.x

Casey, B. J., Giedd, N. J., \& Thomas, K. M. (2000). Structural and Functional Brain Development and its Relation to Cognitive Development. Biological Psychology, 54, 241-257. doi:10.1016/ S0301-0511(00)00058-2

Castelli, D. M., Hillman, C. H., Buck, S. M., \& Erwin, H. (2007). Physical fitness and academic achievement in 3 rd 5 th grade students. Journal of Sport \& Exercise Psychology, 29(2), 239-252.
Chaddock, L., Hillman, C.H. Pontifex, M.B., Johnson, C.R, Raine, L.B., \& Kramer,A.F. (2012). Childhood aerobic fitness predicts cognitive performance one year later. Journal of Sports Sciences, 30(5),421430. doi:10.1080/02640414.2011.647706

Colcombe, S., \& Kramer, A. F. (2003). Fitness Effects on the Cognitive Function of older Adults: a meta-analytical study. Psychological Science, 14(2), 125-130.

Comalli, P. E., Wapner, P., \& Werner, H. (1962). Interference Effects of stroop color-word test in childhood, adulthood, and aging. The Journal os Genetic Psychology, 100, 47-53.

Córdova, C. (2005). TEVA: Programa Computadorizado para Registro e Processamento da Atenção Visual em Investigações com Retardos Mentais Leves. Lectura Educación Física y Deportes, 10(82), 1-5.

Córdova, C., Karnikowski, M., Pandossio, J. E., \& Nobrega, O. T. (2008). Caracterização de Respostas Comportamentais para o Teste de Stroop Computadorizado - TESTINPACS. Neurociências, 4(2), 75-79.

Diamond A. (2000). Close interrelation of motor development and cognitive development and of the cerebellum and prefrontal cortex. Child Development, 71(1),44-56. doi: 10.1111/14678624.00117

Erickson, K. I., Voss, M. W., Prakash, R.S., Basak, C., Szabo, A., Chaddock, L., ... Kramer, A. F. (2011). Exercise training increases size of hippocampus and improves memory. PNAS, 15, 108(7), 3017-3022. doi:10.1073/pnas.1015950108

Gallahue, D.L, \& Ozmun, J. (2005). Compreendendo o Desenvolvimento Motor ( $3^{\mathrm{a}}$ ed.). São Paulo: Phorte.

Gathercole, S. E. The development of memory. (1998). Journal of Child Psychology and Psychiatry, 39(1), 3-27. doi: 10.1111/14697610.00301

Georgopoulos, A. (2000). Neural aspects of cognitive motor control. Neurobiology, 10(2), 238-241. doi: 10.1016/S0959-4388(00)00072-6

Goulardins, J. B., Marques, J. C., Casella, E. B., Nascimento, R., \& Oliveira, J. A. (2013). Motor profile of children with attention deficit hype- 
ractivity disorder, combined type. Research in Developmental Disabilities, 34(1), 40-45. doi: 10.1016/j.ridd.2012.07.014

Hillman, C. H., Castelli, D. M., \& Buck, S. M. (2005). Aerobic Fitness and Neurocognitive Function in Healthy Preadolescent Children. Medicine \& Science in Sport \& Science, 37(11), 1967-1974. doi: 10.1249/01.mss.0000176680.79702.ce

Hillman, C. H., Pontifex, M. B., Raine, L. B, Castelli, D. M., Hall, E. E., \& Kramer, A. (2009). The effect of acute treadmill walking on cognitive control an academic achievement in preadolescent children. Neuroscience, 159(3), 1044-1054. doi: 10.1016/j.neuroscience.2009.01.057

Ladewig, I. (2000). A importância da atenção na aprendizagem de habilidades motoras. Revista Paulista de Educação Física, suplemento 3, 62-71.

Le Boulch, J. (1998). Educação Psicomotora: a psicocinética na idade escolar. Porto Alegre: Artes Médicas.

Medina-Papst, J, Marques, I. Avaliação do desenvolvimento motor de crianças com dificuldades de aprendizagem (2010). Revista Brasileira de Cineantropometria e Desempenho Humano, 12 (1), 36-42.

Middleton, L., Manini, T. M., Simonsick, E. M., Harris, T. B., Deborah E Barnes, D. E., \& Frances, T. et al. (2011) Activity energy expenditure and incident cognitive impairment in older adults. Archives of Internal Medicine, 171(14), 1251-1257. doi:10.1001/archinternmed.2011.277

Pellegrini, A. M., Neto Souza, S., Alleoni, B. N., Gama, D. T., Tinos, S. H., Hatore, R. S., ... \& Motta, A. I. (2003). A aquisição de habilidades motoras a partir de estruturas rítmicas na educação infantil e no ensino fundamental Núcleo de Ensino. São Paulo: Editora UNESP, 9, 35-41.

Piaget, J. (1978). A Construção do Real na Criança. Trad. Álvaro Cabral. Rio de Janeiro: Zahar.

Pontifex, M. B., Scudder, M. R., Brown, M. L., O’Leary, K. C., Wu, C. W., Themanson, J. R., \& Hillman, C. H. (2010). On the number of trials necessary for stabilization of error-related brain activity across the life span. Psychophysiology, 1-7. doi: 10.1111/j.1469-8986.2010.00974.x

Rigoli, D., Piek, J. P., Kane, R., Oosterlaan, J. (2012).
Motor coordination, working memory, and academic achievement in a normative adolescent sample: testing a mediation model. Archives of Clinical Neuropsychology, 27(7), 766-780. doi: 10.1093/arclin/acs061

Romine, C. B., \& Reynolds, C. R. (2004). Senquential Memory: a developmental perspective on its relation to frontal lobe functioning. Neuropsychology Review, 14(1), 43-64. doi: 10.1023/B:NERV. 0000026648.94811 .32

Rosa Neto, F. (2001). Manual de Avaliação Motora. Porto Alegre: Editora Artmed.

Santos, S., Dantas, L., \& Oliveira, J. A. (2004). Desenvolvimento motor de crianças, de idosos e de pessoas com transtornos da coordenação. Revista Paulista de Educação Física, 18, 33-44.

Senra, C. B. N. (2007) A estimulação psicomotora aquática e o desenvolvimento social da criança em idade escolar: eu quero, eu posso, eu escolho, eu coopero. Brasília 2007. (Dissertação) Mestrado em Educação Física. Universidade Católica de Brasília.

Souza Neto, S., Micotti, M. C. O., Benites, L. C., Silveira C. R., \& Alves, C. (2005). A pedagogia do movimento humano - o corpo como objeto de estudo projeto leitura e escrita: a avaliação motora (Ed.). Núcleo de Ensino. São Paulo: Editora UNESP.

Sternberg, R. J. (2000). Psicologia cognitiva. Porto Alegre, RS: Artes Médicas.

Tanner, J. M. (1962). Growth at adolescence (2a ed.). Oxford: Blackwell Scientific.

Tomporowski, P. (2003). Effects of acute bouts of exercise on cognition. Acta Psychologica, 112(3), 297-324. doi: 10.1016/S0001-6918(02)00134-8

Voss, M. W., Chaddock, L., Kim, J. S., Vanpatter, M., Pontifex, M. B., Raine, L. B., ... Kramer, A. F. (2011). Aerobic Fitness Is Associated with Greater Efficiency of the Network Underlying Cognitive Control in Preadolescent Children. Neuroscience, 199, 166-176. doi: 10.1016/j. neuroscience.2011.10.009

Wassenberg, R., Kessels, A. G. H., Kalff, A. C., Hurks, P. P. M., Jplles, J., Feron, F. J. M., ... Vles, J. S. H. (2005). Relation between cognitive and motor performance in 5-to 6 -year-old chil- 
dren: results from a large-scale cross-sectional

study. Child Development, 76(5), 1092-1103. doi:

10.1111/j.1467-8624.2005.00899.x

(cc) EY-NG Todo o conteúdo da revista Motricidade está licenciado sob a Creative Commons, exceto quando especificado em contrário e nos conteúdos retirados de outras fontes bibliográficas. 
